\title{
Modulation of Transient Receptor Potential Channels 3 and 6 Regulates Osteoclast Function with Impact on Trabecular Bone Loss
}

\author{
Sebastian Klein ${ }^{1,5} \cdot$ Birgit Mentrup $^{1} \cdot$ Melanie Timmen ${ }^{1} \cdot$ Joanna Sherwood ${ }^{1} \cdot$ Otto Lindemann $^{2} \cdot$ Manfred Fobker $^{3}$. \\ Daniel Kronenberg ${ }^{1} \cdot$ Thomas Pap $^{1} \cdot$ Michael J. Raschke ${ }^{4} \cdot$ Richard Stange $^{1} \mathbb{D}$
}

Received: 19 November 2019 / Accepted: 8 February 2020 / Published online: 5 March 2020

(c) The Author(s) 2020

\begin{abstract}
Enhanced osteoclast formation and function is a fundamental cause of alterations to bone structure and plays an important role in several diseases impairing bone quality. Recent work revealed that TRP calcium channels 3 and 6 might play a special role in this context. By analyzing the bone phenotype of TRPC6-deficient mice we detected a regulatory effect of TRPC3 on osteoclast function. These mice exhibit a significant decrease in bone volume per tissue volume, trabecular thickness and -number together with an increased number of osteoclasts found on the surface of trabecular bone. Primary bone marrow mononuclear cells from TRPC6-deficient mice showed enhanced osteoclastic differentiation and resorptive activity. This was confirmed in vitro by using TRPC6-deficient RAW 264.7 cells. TRPC6 deficiency led to an increase of TRPC3 in osteoclasts, suggesting that TRPC3 overcompensates for the loss of TRPC6. Raised intracellular calcium levels led to enhanced NFAT-luciferase reporter gene activity in the absence of TRPC6. In line with these findings inhibition of TRPC3 using the specific inhibitor Pyr3 significantly reduced intracellular calcium concentrations and normalized osteoclastic differentiation and resorptive activity of TRPC6-deficient cells. Interestingly, an up-regulation of TRPC3 could be detected in a cohort of patients with low bone mineral density by comparing micro array data sets of circulating human osteoclast precursor cells to those from patients with high bone mineral density, suggesting a noticeable contribution of TRP calcium channels on bone quality. These observations demonstrate a novel regulatory function of TRPC channels in the process of osteoclastic differentiation and bone loss.
\end{abstract}

Keywords Transient receptor potential channels $\cdot$ TRPC $\cdot$ Osteoporosis $\cdot$ Osteoclast $\cdot$ Bone resorption $\cdot$ Calcium signaling

Sebastian Klein and Birgit Mentrup have contributed equally to this work.

Electronic supplementary material The online version of this article (https://doi.org/10.1007/s00223-020-00673-8) contains supplementary material, which is available to authorized users.

Richard Stange

Richard.Stange@ukmuenster.de

1 Institute of Musculoskeletal Medicine, University Münster, Münster, Germany

2 Institute of Physiology II, University Münster, Münster, Germany

3 Center for Laboratory Medicine, University Hospital Münster, Münster, Germany

4 Department of Trauma, Hand and Reconstructive Surgery University Hospital Münster, Münster, Germany

5 Institute of Pathology, University Hospital of Cologne, Cologne, Germany

\section{Introduction}

Human diseases with an acquired loss of bone account for increased mortality especially among elderly men and women [1]. A number of loci associated with bone mineral density and fracture risk have been identified by genomewide association studies [2]. Nevertheless, uncovering novel genes and pathways regulating bone metabolism is a key in providing the basis for new therapeutic options to prevent bone loss and subsequent fractures.

On the cellular level, monocyte/macrophage derived osteoclasts are considered to mediate the exceeding loss of bone. Their differentiation depends on the availability of intracellular calcium ions, since calcium binding stimulates phosphatases including calcineurin [3], which subsequently facilitates the translocation of factors such as nuclear factor of activated T-cells NFATc1 into the nucleus and induces the transcription of osteoclastogenesis-promoting genes [4]. 
Many different membrane channels, including the transient receptor potential (TRP) ion channel family, control the entry of calcium ions into the cells [5]. This family consists of six subgroups including the canonical (TRPC) and the vanilloid (TRPV) subtype, with TRPV channels previously demonstrated as modulators of calcium signaling in osteoclasts [6, 7]. TRPC channels belong to a group of seven cation channels divided into subgroups according to their amino acid sequence similarities and they can assemble as homo- and heterotetramers [8].

The subgroup of TRPC $3 / 6 / 7$ channels share between 70 and $80 \%$ sequence homology; however, they demonstrate distinctly different affinities for calcium cations. TRPC3 and TRPC7 functionally regulate basal calcium influx, whereas TRPC6 has been described as a receptor operated calcium channel $[9,10]$.

TRPC 3 and TRPC6 channels have already been targeted in pre-clinical models of renal- and cardiac fibrosis, as well as in the treatment of hypoxic vasoconstriction [11, 12]. A role of TRPC6 in the pathogenesis of certain kidney diseases has been described, where gain-of-function mutations lead to increased podocyte apoptosis due to altered NFAT signaling [13, 14]. It is believed that TRPC7 also plays a role in calcium signaling through its characteristic features, but its physiological importance in the native environment is not yet clear [15].

Although TRPC3 and TRPC6 have been investigated in murine and human osteoblast-like cells [16], the functional role of these TRPC channels in osteoclasts has not been previously clarified. Therefore, in this study, we aimed to examine whether TRPC 3 and TRPC6 channels are involved in bone loss and regulation of osteoclast formation and function via $\mathrm{Ca}^{2+}$ and NFAT signaling and whether modulation of TRPCs 3 and 6 may effect bone homeostasis.

\section{Materials and Methods}

\section{Transcriptome Analysis}

The publicly available data sets GSE7158 and GSE56815 (https://www.ncbi.nlm.nih.gov/geo/), basing on micro array analyses of circulating monocytes, were evaluated for TRPC channel expression, using R (the R-project, https://cran.rproject.org). Data sets GSE7158 and GSE56815 both compare human individuals with high and low bone mineral density (BMD), respectively [17]. Gene names of array IDs were assigned corresponding to 'https://www.ensembl.org/ biomart/martview/'. The following free-available R-packages were used: 'affy', 'affyQCReport', 'lattice', 'limma', 'tkWidgets', 'widgetTools', 'tcltk', 'DynDoc', 'oligo', 'gplot' (https://www.bioconductor.org). The corresponding genes were plotted after z-score transformation for each gene.

\section{Animals}

TRPC $^{+/-}$mice [18] were kindly provided by the group of Alexander Dietrich from Munich, Germany. Animals were housed in a temperature-controlled animal room with water and conventional lab chow ad libitum and with a $12 / 12 \mathrm{~h}$ light/dark cycle. Heterozygous mice were used for breeding and only female littermates of TRPC6 ${ }^{-/-}$and WT background were used for the experiments. Details of serum and urine analyses are provided in the supplements.

\section{$\mu C T$ Analysis}

Bone-structures of 4 month old mice were analyzed by micro-computed tomography $(\mu \mathrm{CT})$ on a Bruker Skyscan 1176 in vivo microCT (Kontich, Belgium). The X-ray tube was set to $45 \mathrm{kVp}$, and the voxel size was $9 \mathrm{~mm}^{3}$. Images were reconstructed and analyzed using manufacturer's software (NRecon, CTAn, CTVol dataviewer; Bruker microCT). The total bone volume fraction (the percentage of bone volume to total volume, BV/TV), mean trabecular thickness (Tb.Th), trabecular separation (Tb.Sp), and trabecular number (Tb.N) were calculated for trabecular bone, whereas the average cortical thickness (Ct.Th) was quantified from cortical bone.

\section{Cell Culture}

Details of cell isolation, culture, differentiation, staining and expression analyses are provided in the supplementary materials and methods.

\section{Generation Of TRPC6 Deficient RAW 264.7 Cells By CRISPR/Cas9 Technology}

For cellular knock out of TRPC6 in RAW 264.7 cells sgRNAs were designed, targeting exon 2 and comprising all transcript variants of TRPC6 in mice. For subcloning into lentiCRISPRv2 [19] (Addgene \#52,961) the sequence was modified by attachment of BsmBI restriction sites. One Shot Stbl3 Escherichia coli (Invitrogen, Thermo Fisher Scientific) were transformed and the correct insertion of the sgRNA was verified by sequencing. For production of lentivirus HEK293T cells were transfected with lentiCRISPRv2_sgRNA29, psPAX2 (Addgene \#12,260) and pMD2.G (Addgene \#12,259) at the ratio of 4:3:1 by Lipofectamine 2000 according to the manufacturer's protocol (Invitrogen, Thermo Fisher Scientific). Medium was replaced after 12 h; supernatants were harvested after $24 \mathrm{~h}$ and $48 \mathrm{~h}$, and filtered through a $0.22 \mu \mathrm{m}$ membrane. RAW 264.7 cells were seeded in 24 well plates at a density of $10^{4}$ cells/well and 
incubated with lentiviruses in the presence of $8 \mu \mathrm{g} / \mathrm{mL}$ polybrene (Sigma-Aldrich). Transduced RAW 264.7 cells were positively selected with puromycin (Gibco, Thermo Fisher Scientific), followed by clonal expansion using a serial dilution assay. DNA was isolated from clonally derived cells, amplified with 5' and $3^{\prime}$ flanking primers located in intron 1 (Pr_003-for) and exon 2 (Pr_587-rev), followed by sequence analysis using a sequencing service (Eurofins Genomics, Ebersberg, Germany). Successful knock out was validated by alignment of DNA sequences with wild type sequences.

\section{Intracellular $\mathrm{Ca}^{2+}$ Measurements}

Five $\times 10^{3}$ RAW 264.7 cells/well were seeded in 96 well plates. One day before incubation with $3 \mu \mathrm{M}$ Fura-2AM (ThermoFisher Scientific) for $1 \mathrm{~h}$ at $37^{\circ} \mathrm{C}$, cells were costimulated with $100 \mathrm{ng} / \mathrm{mL}$ RANKL and $10 \mu \mathrm{M}$ Pyr3, or solvent control, respectively [20]. After three washes with Ringer's solution at RT, measurements were performed at $24{ }^{\circ} \mathrm{C}$ for $150 \mathrm{~s}$ on a Spark $10 \mathrm{M}$ microplate reader (TECAN). The change in intracellular calcium $\left(\mathrm{Ca}^{2+}\right)$ concentration was indicated as the ratio of fura- 2 fluorescence intensities for $340 \mathrm{~nm}\left(\mathrm{Ca}^{2+}\right.$ bound to fura-2) and $380 \mathrm{~nm}$ (free fura-2) excitation (F ratio), at an emission wavelength of $510 \mathrm{~nm}$.

\section{Statistical Analysis}

Statistical analyses were performed using R, the R-project as well as GraphPad Prism v6 and v8 (GraphPad Software, Inc, La Jolla, CA USA). Due to the small sample sizes, we used the Mann-Whitney $U$ test for differences. All data are reported as mean standard deviation (SD). A value of $p<0.05$ was considered as statistically significant.

\section{Results}

\section{TRPC6 Deficient Mice Show an Impaired Bone Structure}

The analysis of the fifth lumbar vertebrae of 4-month old female mice by $\mu \mathrm{CT}$ demonstrated an osteoporosis-like bone phenotype in TRPC $^{-1-}$ mice $(n=7)$ when compared to wild type littermates $(n=10)$ (Fig. 1a). The bone volume divided by tissue volume (BV/TV) was significantly decreased in TRPC $^{-1-}$ mice. In addition, these mice revealed decreased trabecular number, together with decreased trabecular thickness. No significant differences could be detected with regard to cortical thickness (C.Th) or trabecular separation (Tb.Sp), respectively. When comparing bone length (tibia) of 8 week- and 16 week old wild type mice and TRPC $6^{-1-}$ littermates and vertebral height and width of 16 week old animals we could not find any difference between the genotypes (Fig. S1). Quantification of osteoclasts in tissue sections of lumbar vertebrae showed an increase in osteoclast number in $\mathrm{TRPC}^{-/}$mice. The number of osteoclasts per trabecular area (N.OC./T.A), the average bone surface covered by osteoclasts (Oc.S/BS), as well as the average size of osteoclasts (Oc.S/N.Oc) were elevated. No differences for the number of osteoblasts were observed (Fig. 1b). Quantification of C-terminal collagen X (CTX) as a serum marker of bone resorption, revealed $1.37 \mathrm{ng} / \mathrm{mL}$ in wild type animals $(n=7)$ compared to $3.06 \mathrm{ng} / \mathrm{mL}$ in TRPC6 ${ }^{-1-}$ mice $(n=6)$. Representative reconstructed microCT pictures and images of TRAP-stained vertebrae of WT and TRPC $6^{-1-}$ mice are presented in Fig. 1c. The analyses of urine and serum markers for calcium metabolism showed no differences between these genotypes regarding calcium, phosphate and creatinine levels, respectively (Fig. S2).

\section{TRPC6 Ablation Enhances TRPC3 Expression, Osteoclast Differentiation and Bone Resorption}

Quantitative RT-PCR analyses showed relatively low basal expression of TRPC 3 and TRPC6 in primary osteoclast precursor cells from WT mice (Fig. 2a). During M-CSF and RANKL stimulation expression levels of both calcium channels increased. In more detail, highest expression was observed on day 2; on day 4 the TRPC 3 expression level stagnated, whereas the TRPC6 level was slightly reduced. In cells from TRPC6 $^{-1-}$ mice expression of TRPC 3 was induced 3.8 fold on day 2 of osteoclastic differentiation, the difference compared to WT was significant on day 4 . A successful osteoclastogenesis was proven by an increased expression of marker genes cathepsin K, SIRP $\alpha$ and MMP9 (Fig. S3).

To further confirm the data obtained from primary cells, RAW 264.7 cells were manipulated by CRISPR/Cas9technology to knock out the TRPC6 gene. The analyses of genomic DNA from lentiviral transduced RAW 264.7 cells after selection and clonal expansion revealed a deduced truncated TRPC6 protein with a stop codon after amino acid (aa) position 112 and a deletion of 41 aa from position 74 to 114 on the other allele (Fig. S4). This deleted region is located in the first ankyrin repeat, which is an accepted motif for homo-/heterodimerization [21].

Immunocytochemical staining of fixed RAW 264.7 cells confirmed a considerably increased expression of TRPC3 in the absence of TRPC6 (Fig. 2b). Although we performed control staining with TRPC6 antibodies from different manufacturers under various experimental conditions, we got signals in cells from TRPC6 ${ }^{-/-}$knock out mice and cell line with these antibodies, probably because of the way the knock out was generated (Fig. S5). 


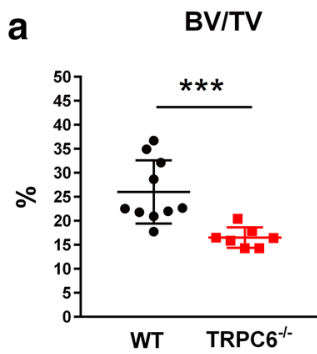

Tb.N.

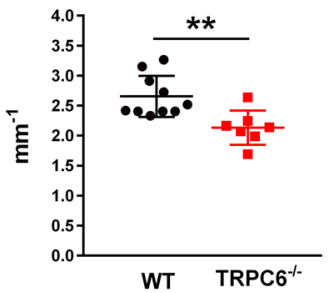

b

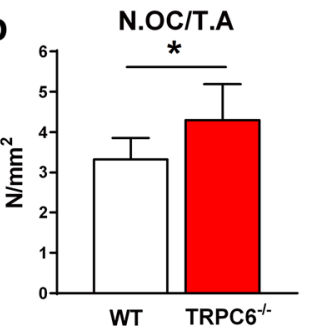

Oc.S/BS
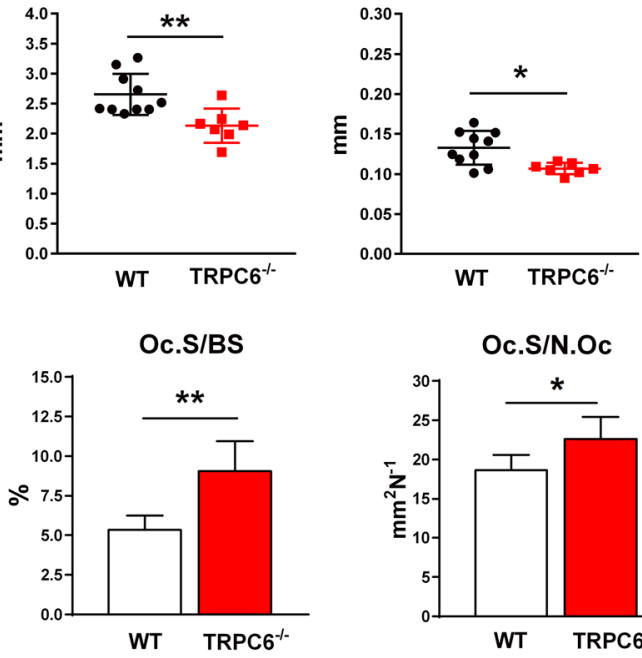

C

WT
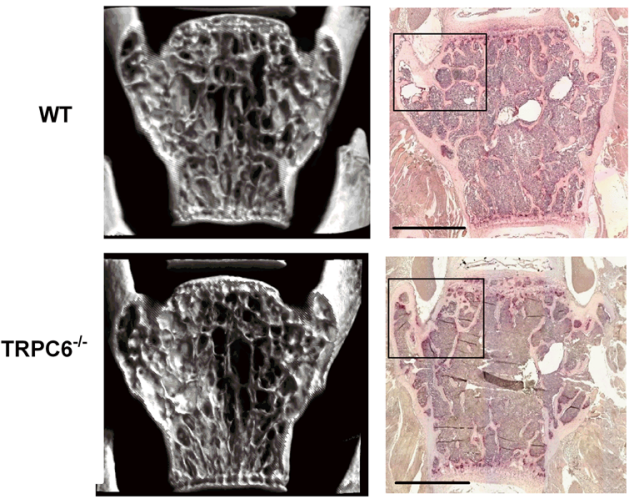

Tb.Th.

C.Th.

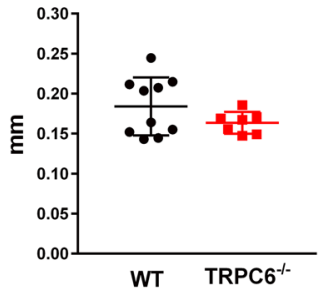

Oc.S/N.OC
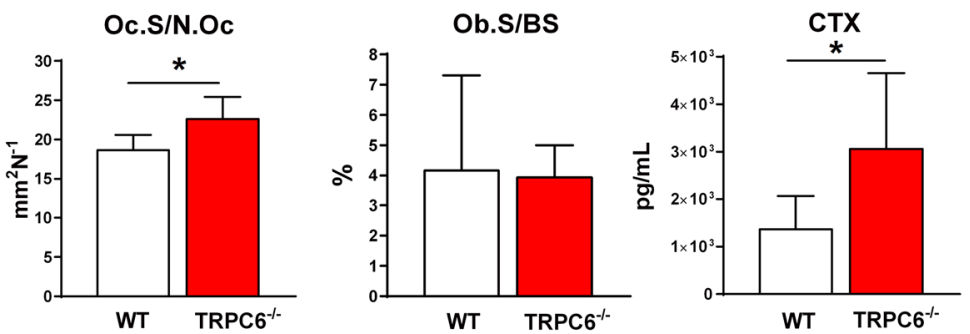

Fig. 1 TRPC6 deficiency points to bone loss. a MicroCT analyses of the fifth lumbar vertebrae revealed an osteoporosis-like phenotype in $\mathrm{TRPC}^{-/-}$mice compared to age-matched wild type littermates. b Quantification of osteoclasts in tissue sections showed an increase in osteoclast number and size in $\mathrm{TRPC}^{-/-}$mice. No differences regarding osteoblasts were detected, but elevated CTX serum marker.
$* p<0.05 ; * * p<0.01 ; * * * p<0.001$. c Representative reconstructed $\mu \mathrm{CT}$ pictures of the fifth lumbar vertebrae and representative images of TRAP-stained vertebrae of WT and TRPC $6^{-/}$mice, counterstained with Hematoxylin. Arrows indicate strong osteoclast staining. Scale bars $=1 \mathrm{~mm}$

\section{Inhibition of TRPC3 Abrogates Enhanced Intracellular Calcium Levels and Increased Bone Resorption}

Measurement of intracellular calcium concentrations revealed an approximately $10 \%$ higher ratio of $\mathrm{Ca}^{2+}$-bound to free fura-2 in TRPC6-deficient RANKLstimulated RAW 264.7 cells, compared to RAW 264.7 WT cells (Fig. 3a). Blocking TRPC 3 using the specific inhibitor Pyr3 decreased this ratio to approximately $90 \%$ 
a
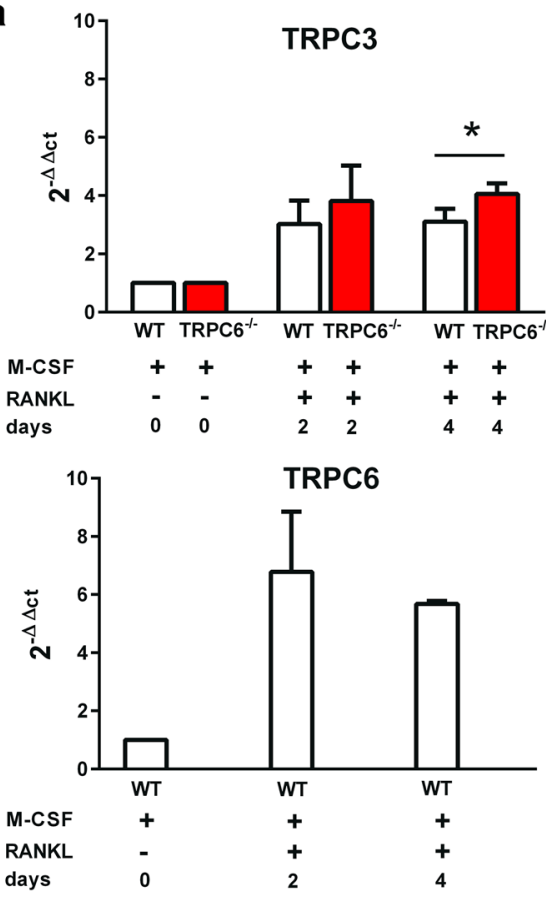

C
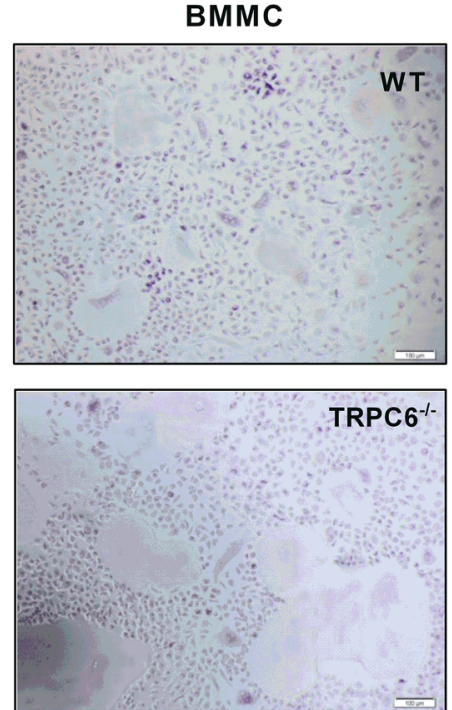

b

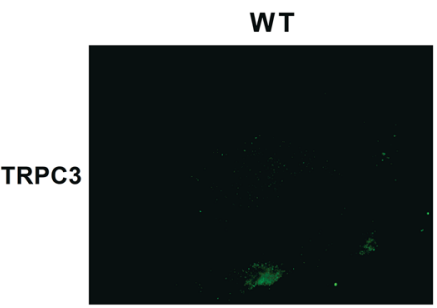

RAW 264.7

WT

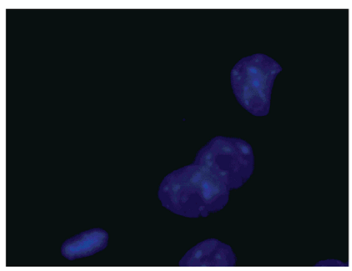

DAPI
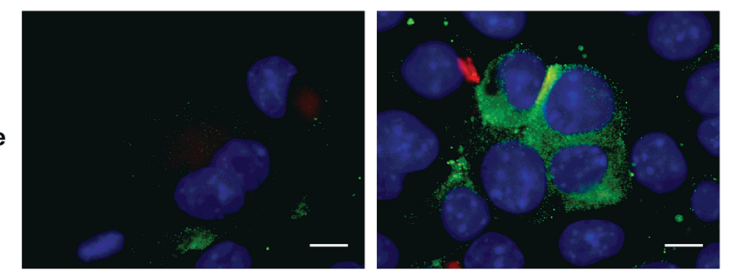

BMMC

d
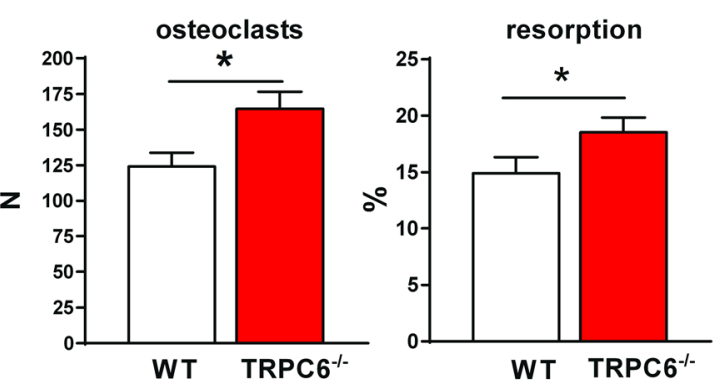

e

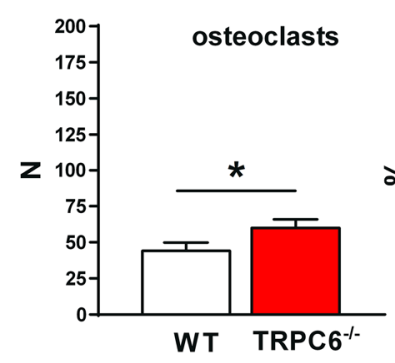

RAW 264.7

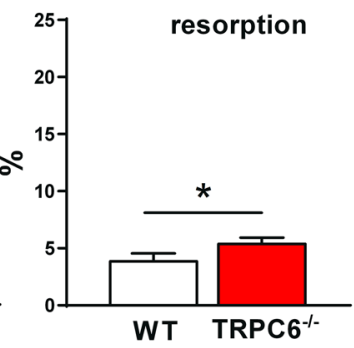

Fig. 2 TRPC6 deficiency results in enhanced TRPC3 expression in osteoclasts. a Expression of TRPC3 and TRPC6 increases during osteoclastogenesis and TRPC3 is significantly higher expressed in cells from $\mathrm{TRPC}^{-/-}$mice. Data are averages $\pm \mathrm{SD}(n=3), p$-value determined by Mann-Whitney $\mathrm{U}$ test, two-tailed test, $p<0.05$. b Imunocytochemical staining of WT and TRPC6 $^{-1-}$ RAW 264.7

in both WT and TRPC6-deficient RAW 264.7 cells, respectively. cells confirms considerably enhanced TRPC3 expression in TRPC6deficient cells (scale bars $=10 \mu \mathrm{m}$ ). c Size of differentiated BMMC (scale bars $=100 \mu \mathrm{m}$ ) and number as well as resorptive activity of osteoclasts is significantly elevated in TRPC6 $^{-/-} \mathbf{d ~ B M M C}$ and e RAW264.7 cells. * $p \leq 0.05$

The impact of intracellular calcium concentrations on nuclear factor of activated T-cells (NFAT) promoter activity was determined by luciferase reporter gene assay. The assay 

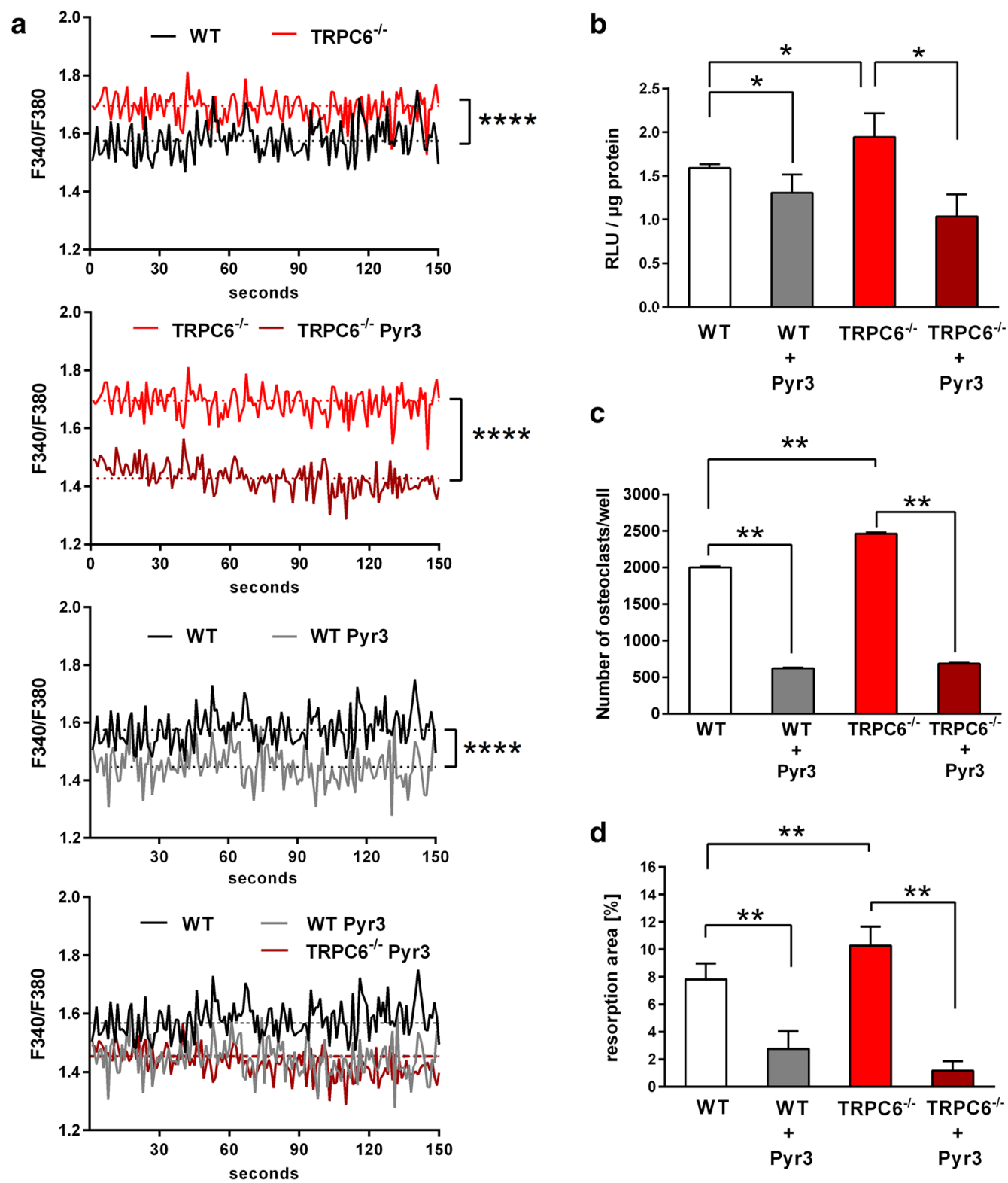

Fig. 3 TRPC3 inhibition can reverses the effects of TRPC6 deficiency. a Representative comparative measurement of intracellular calcium, illustrated by $\mathrm{F} 340 / \mathrm{F} 380$ ratios, show significant higher levels in TRPC6 $^{-1-}$ RAW 264.7 cells than in WT cells and effective reduction of intracellular calcium by Pyr3-inhibition of TRPC3 $(* * * * p<0.0001)$. b NFATc1 promoter activity is increased in TRPC6

revealed significantly increased relative light units (RLU)/ $\mu \mathrm{g}$ protein in transfected TRPC6-deficient RANKL-stimulated RAW 264.7, compared to RAW 264.7 WT cells (Fig. 3b). Simultaneous stimulation with Pyr3 significantly decreased this value in TRPC6-deficient RAW 264.7 cells and in RAW 264.7 WT cells.

The inhibitory effect of Pyr3 on TRPC 3 was also reflected on number and resorptive activity of osteoclasts from differentiated primary $\mathrm{BMMC}$ of $\mathrm{TRPC}^{-/-}$mice deficient RAW264.7 cells and can be significantly reduced by Pyr3 in both, WT and TRPC6 deficient RAW 264.7 cells, respectively $(n=3 ; p<0.05)$. c The effect is reflected with the same pattern by the counted number of TRAP-stained osteoclasts $(p<0.01)$ and $\mathbf{d}$ by evaluating the percentage resorption area $(p<0.01)$

and WT mice. Inhibition of TRPC 3 reduced the general number of osteoclasts in cells from $\mathrm{TRPC}^{-1-}$ mice, and in cells from WT mice, respectively (Fig. 3c). The relative resorption area determined by area fraction $(n=8)$, was increased in TRPC6 $6^{-1-}$ compared to WT, and was clearly decreased in both, Pyr3-treated TRPC6 ${ }^{-1-}$ cells and Pyr3-treated WT cells. By comparing WT cells and $\mathrm{TRPC}^{-/-}$cells in the presence of TRPC3 inhibitor Pyr3, 

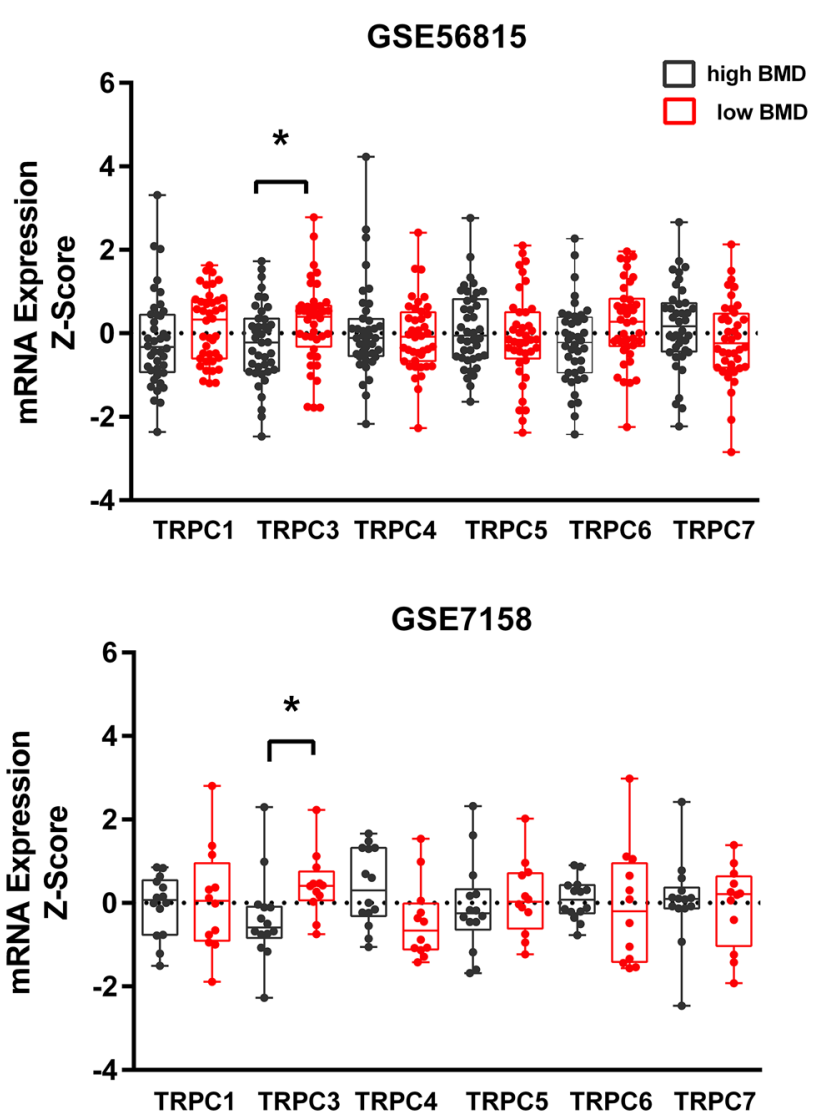

Fig. 4 TRPC channel expression in human individuals. TRPC3 channel expression in human circulating monocytes is significantly higher in individuals with lower bone-mineral-density (BMD). GSE56815 $n=80 ;$ GSE7158 $n=26 ;{ }^{*} p<0.05$

we observe even a little stronger effect of TRPC 3 inhibition on TRPC6-deficient cells (Fig. 3d).

\section{TRPC3 Expression is Upregulated in Human Individuals with Low Bone Mineral Density}

To elucidate the role of TRPC expression in acquired bone loss in a human population, we analyzed two publicly available data sets from a transcriptome-wide expression study and uncovered a putative relationship regarding the expression levels of TRPC channels in human osteoclast precursor cells of individuals with low BMD and high BMD [17] (Fig. 4). The data set GSE56815, a Caucasian cohort, revealed a significantly higher expression of TRPC3 in 40 females [age 50.0 (SD 7.9) years] with an average hip BMD Z score of - 1.05 (SD 0.44) when compared to 40 females [age 49.4 (8.1) years] with an average hip BMD Z score of 1.45 (0.67). In addition, a significant increase in TRPC3 expression could be detected by comparing data set GSE7158, an Asian cohort of 12 females [age 25.3 (3.1) years] with an average hip BMD Z score of $-1.72(0.60)$ and 14 females [age 28.7 (4.7) years] with a hip BMD Z score of $1.57(0.57)$ $(p<0.05)$.

\section{Discussion}

We show-for the first time-that modulation of the transient receptor potential cation channels TRPC 3 and TRPC6 has consequences for the quality of bone. Our transcriptome analysis revealed that women with low bone mineral density show an increased expression of TRPC 3 in human osteoclast precursor cells. In mice we measured increased TRPC3 expression after ablation of TRPC6, resulting in an altered bone structure, leading to a phenotype found in osteoporosis. An increased activation of osteoclasts both in vivo and in vitro could be confirmed. In detail, mice lacking TRPC6 revealed decreased bone volume per tissue volume in the lumbar vertebrae, as well as decreased trabecular thickness and trabecular number. Accordingly, number and size of osteoclasts on the surface of trabecular bone and the level of the biomarker CTX in the serum of these mice were significantly increased.

Ong et al. [22] reported an involvement of TRPC1 in osteoclast formation and function, but so far, there is no direct evidence to suggest a particular role of TRPC 3 or TRPC6 channels in bone metabolism. We detected low basal expression for both channels on osteoclast precursor cells, which increased during osteoclastogenesis. Comparison of primary BMMCs isolated from TRPC6 knock out mice and WT mice showed an increased osteoclastic differentiation capacity along with elevated bone resorption activity of TRPC6 deficient cells. This effect is not a phenomenon limited to ablation of TRPC6 in the germline; it could also be confirmed in RAW 264.7 cells after disruption of TRPC6 by CRISPR/Cas9.

The expression of TRPC3 was increased in TRPC6 deficient osteoclasts, compared to differentiated cells from wild type littermates. This is in accordance with previous findings in vascular smooth muscle cells of TRPC6 deficient mice [18]. Osteoclast function might rely on an equilibrium between TRPC 3 and TRPC6, leading to bone loss in mice lacking TRPC6. Recent studies showed discrepancies regarding expression and functional contribution of these channels [23, 24], which may be related to the fact that the contribution of TRPC 3 and TRPC6 channels depends on their association as homo- or heterotetrameric complexes and differences in their association pattern may change their function [25].

It is known from previous studies, that calcium signaling in osteoclasts plays a vital role in their differentiation process, but there is only limited data available on the regulation of calcium signaling in these cells $[4,26]$. Given that TRPC 3 and TRPC6 channels have different affinities to cations [27], 
we demonstrated that basal intracellular calcium is elevated in TRPC6 deficient osteoclast precursor cells. Dietrich et al. [18] have initially described this phenomenon; they showed increased expression levels of TRPC3 in smooth muscle cells of blood vessels after ablation of TRPC6. The up-regulation of TRPC3 after loss of TRPC6 has been reported as being rather cell-type specific [28]. While the molecular mechanism of these findings remains to be understood, one may consider a possible feed-forward loop of TRPC3 expression that is coupled to increased influx of calcium.

TRPC6 is a cation channel that depends on receptor activation, and absence of TRPC6 gives rise to TRPC3-only composites that show basal channel activity. Because inhibition of TRPC3 using Pyr3 can diminish increased intracellular calcium levels, elevated differentiation, and increased bone resorption of TRPC6 deficient osteoclasts, we propose that elevated TRPC3 presence is driving the increased activity of osteoclasts when TRPC6 is missing. Indeed, TRPC3 expression has been linked to bone biology on several occasions. Our data analyses demonstrate that human individuals with low BMD have a significantly increased expression of TRPC3 in circulating monocytes, the precursors of osteoclasts, when compared to individuals with high BMD. A further indication that elevated TRPC3 expression might be related to increased bone resorption can be concluded from the Williams-Beuren Syndrome, a neurodevelopmental disorder where osteopenia is a common feature of the disease. Increased levels of TRPC3 channels have been described on somatic cells of an individual [29, 30]. Moreover, recent evidence suggests a TNF- $\alpha$ dependent induction of TRPC3, outlining this channel as constitutively active, and participating in NF-kB signaling [31]. This supports a role of TRPC3 in regulating pathways that are well known to drive differentiation and resorption activity of osteoclasts [31, 32]. Furthermore Becker et al. [33] characterized the moonwalker (mwk) mouse, which has a dominant T635A TRPC3 gainof-function mutation. These mice have an abnormal Purkinje cell development and cerebellar ataxia. Interestingly, DEXA scans of heterozygous mwk mice showed significant lower bone mineral densities compared to wild type mice (www. mousephenotype.org/data/genes/MGI:109526), which supports the hypothesis, that an increased activity of TRPC3 is disadvantageous for a healthy bone.

These findings recommend TRPC3 as an interesting target to modulate bone metabolism. Osteoporosis in elderly men and women, together with secondary bone loss in diseases including cancer, pose a major risk factor for fractures, immobilization and hospitalization [34, 35]. Targeting osteoclastic bone resorption lowers the risk of fractures and improves the outcome of patients suffering from bone loss [36, 37].

A variety of medications that suppress bone resorption by affecting the activity of osteoclasts have been developed so far [38, 39]. Despite their good efficiency in increasing bone mass, these drugs are associated with a number of side effects that limit their therapeutic application in a subgroup of patients that would benefit from an intervention [40, 41]. TRPC 3 is already in the focus of interest as a target for pharmacological interventions due to the observation that gain of function is associated with pathologies of the cardiovascular system and the brain [42]. A suitability for an application of the pyrazole 3 (Pyr3) developed by Kiyonaka et al. to inhibit TRPC3 in vivo has already been shown [43].

We could demonstrate that the increased intracellular calcium levels after ablation of TRPC6 are abolished by inhibition of TRPC3 using Pyr3. Hence, we conclude that TRPC3 is regulating influx of calcium from extracellular compartments in osteoclasts. These findings underscore a regulatory function of TRPC on osteoclasts.

In summary, we report that (i) loss of the cation channel TRPC6 in vivo leads to an osteoporosis-like phenotype due to increased size and activity of osteoclasts; (ii) ablation of TRPC6 in osteoclasts leads to increased expression of TRPC3, resulting in elevated intracellular calcium, differentiation, and resorption activity; (iii) inhibition of TRPC3 lowers bone resorption and decreases differentiation of osteoclasts.

Based on these data, we propose that TRPC3 represents a potent driver of osteoclastogenesis, which is regulated by the presence of TRPC6.

Acknowledgements Open Access funding provided by Projekt DEAL. We would like to thank I. Leifert and S. Niehues for great technical assistance.

Author Contributions S.K. participated in project planning, designed and performed experiments and wrote the manuscript; B.M. performed gDNA sequencing and immunocytochemistry, and participated in data analysis, interpretation and manuscript preparation; M.T. participated in project planning, data analysis, interpretation and manuscript preparation; O.L. gave advice on calcium measurements; M.F. performed the measurements of calcium metabolism; D.K. performed $\mu \mathrm{CT}$ measurements, data analysis and interpretation and manuscript preparation; T.P., J.S. and M.J.R. participated in data analysis, interpretation and manuscript preparation; R.S. participated in project planning, data analysis, interpretation and manuscript preparation. All authors contributed to the editing of the manuscript.

Funding This work was financially supported by the Elsbeth-BonhoffFoundation for osteoporosis research to R.S. and M.T. (Project 142), as well as by the Else Kröner-Fresenius Stiftung (EKFS-2014-A06 and 2016_Kolleg.19) to S.K. and by 'Cells-in-Motion Cluster of Excellence' (EXC 1003-CiM), University of Münster, Germany to R.S., M.T. and B.M.

\section{Compliance with Ethical Standards}

Conflict of interest Sebastian Klein, Birgit Mentrup, Melanie Timmen, Joanna Sherwood, Otto Lindemann, Manfred Fobker, Daniel Kronenberg, Thomas Pap, Michael J. Raschke, and Richard Stange declare that there is no conflict of interest regarding the publication of this paper. 
Human and Animal Rights Experimental protocols were approved by the local committee 'Landesamt für Natur, Umwelt und Verbraucherschutz Nordrhein-Westfalen (LANUV)' (84-02.05.50.15.005).

Informed Consent Signed informed-consent documents were obtained from all study participants before entering the study.

Open Access This article is licensed under a Creative Commons Attribution 4.0 International License, which permits use, sharing, adaptation, distribution and reproduction in any medium or format, as long as you give appropriate credit to the original author(s) and the source, provide a link to the Creative Commons licence, and indicate if changes were made. The images or other third party material in this article are included in the article's Creative Commons licence, unless indicated otherwise in a credit line to the material. If material is not included in the article's Creative Commons licence and your intended use is not permitted by statutory regulation or exceeds the permitted use, you will need to obtain permission directly from the copyright holder. To view a copy of this licence, visit http://creativecommons.org/licenses/by/4.0/.

\section{References}

1. Bliuc D, Nguyen ND, Nguyen TV, Eisman JA, Center JR (2013) Compound risk of high mortality following osteoporotic fracture and refracture in elderly women and men. J Bone Miner Res 28:2317-2324. https://doi.org/10.1002/jbmr.1968

2. Clark GR, Duncan EL (2015) The genetics of osteoporosis. Br Med Bull 113:73-81. https://doi.org/10.1093/bmb/ldu042

3. Klee CB, Ren H, Wang X (1998) Regulation of the calmodulin-stimulated protein phosphatase, calcineurin. J Biol Chem 273:13367-13370. https://doi.org/10.1074/jbc.273.22.13367

4. Sato K, Suematsu A, Nakashima T, Takemoto-Kimura S, Aoki $\mathrm{K}$, Morishita Y, Asahara $\mathrm{H}$ et al (2006) Regulation of osteoclast differentiation and function by the CaMK-CREB pathway. Nat Med 12:1410-1416. https://doi.org/10.1038/nm1515

5. Clapham DE, Runnels LW, Strübing C (2001) The trp ion channel family. Nat Rev Neurosci 2:387-396. https://doi. org/10.1038/35077544

6. van der Eerden BC, Joost GJ, Hoenderop JG, de Vries TJ, Schoemaker T, Buurman CJ, Uitterlinden AG et al (2005) The epithelial $\mathrm{Ca} 2+$ channel TRPV5 is essential for proper osteoclastic bone resorption. Proc Natl Acad Sci USA 102:17507-17512. https:// doi.org/10.1073/pnas.0505789102

7. Kajiya H, Okamoto F, Nemoto T, Kimachi K, Toh-Goto K, Nakayana S, Okabe K (2010) RANKL-induced TRPV2 expression regulates osteoclastogenesis via calcium oscillations. Cell Calcium 48:260-269. https://doi.org/10.1016/j.ceca.2010.09.010

8. Hofmann T, Schaefer M, Schultz G, Gudermann T (2002) Subunit composition of mammalian transient receptor potential channels in living cells. Proc Natl Acad Sci USA 99:7461-7466. https:// doi.org/10.1073/pnas.102596199

9. Boulay G, Zhu X, Peyton M, Jiang M, Hurst R, Stefani E, Birnbaumer L (1997) Cloning and Expression of a Novel Mammalian Homolog of Drosophila Transient Receptor Potential (Trp) Involved in Calcium Entry Secondary to Activation of Receptors Coupled by the Gq Class of G Protein. J Biol Chem 272:2967229680. https://doi.org/10.1074/jbc.272.47.29672

10. Dietrich A, Mederos Y, Schnitzler M, Emmel J, Kalwa H, Hofmann T, Gudermann T (2003) N-Linked protein glycosylation is a major determinant for basal TRPC3 and TRPC6 channel activity. J Biol Chem 278:47842-47852. https://doi.org/10.1074/jbc.M3029 83200
11. Saliba Y, Karam R, Smayra V, Aftimos G, Abramowitz J, Birnbaumer L, Farès N (2015) Evidence of a role for fibroblast transient receptor Potential canonical $3 \mathrm{Ca}^{2+}$ channel in renal fibrosis. J Am Soc Nephrol 26:1855-1876. https://doi.org/10.1681/ ASN.2014010065

12. Urban N, Hill K, Wang L, Kuebler WM, Schaefer M (2012) Novel pharmacological TRPC inhibitors block hypoxia-induced vasoconstriction. Cell Calcium 51:194-206. https://doi.org/10.1016/j. ceca.2012.01.001

13. Winn MP, Conlon PJ, Lynn KL, Farrington MK, Creazzo T, Hawkins AT, Daskalakis N et al (2005) A mutation in the TRPC6 cation channel causes familial focal segmental glomerulosclerosis. Science 308:1801-1804. https://doi.org/10.1126/science.1106215

14. Chiluiza D, Krishna S, Schumacher VA, Schlöndorff J (2013) Gain-of-function mutations in transient receptor potential C6 (TRPC6) activate extracellular-signal-regulated kinases $1 / 2$ (Erk1/2). J Biol Chem 288:18407-18420. https://doi.org/10.1074/ jbc.M113.463059

15. Numaga-Tomita T, Oda S, Nishiyama K, Tanaka T, Nishimura A, Nishida M (2019) TRPC channels in exercise-mimetic therapy. Pflugers Arch - Eur J Physiol 471:507-517. https://doi. org/10.1007/s00424-018-2211-3

16. Abed E, Labelle D, Martineau C, Loghin A, Moreau R (2009) Expression of transient receptor potential (TRP) channels in human and murine osteoblast-like cells. Mol Membr Biol 26:146158. https://doi.org/10.1080/09687680802612721

17. Liu YZ, Zhou Y, Zhang L, Li J, Tian R, Zhang JD, Deng HW (2015) Attenuated monocyte apoptosis, a new mechanism for osteoporosis suggested by a transcriptome-wide expression study of monocytes. PLoS ONE 10:1-18. https://doi.org/10.1371/journ al.pone. 0116792

18. Dietrich A, Mederos Y, Schnitzler M, Gollasch M, Gross V, Storch U, Dubrovska G et al (2005) Increased vascular smooth muscle contractility in TRPC6 ${ }^{-1-}$ mice. Mol Cell Biol 25:6980-6989. https ://doi.org/10.1128/MCB.25.16.6980-6989.2005

19. Sanjana NE, Shalem O, Zhang F (2014) Improved vectors and genome-wide libraries for CRISPR screening. Nat Methods 11:783-784. https://doi.org/10.1038/nmeth.3047

20. Schleifer H, Doleschal B, Lichtenegger M, Oppenrieder R, Derler I, Frischauf I, Glasnov TN et al (2012) Novel pyrazole compounds for pharmacological discrimination between receptor-operated and store-operated $\mathrm{Ca}^{(2+)}$ entry pathways. Br J Pharmacol 167:1712-1722. https://doi.org/10.1111/j.1476-5381.2012.02126 . $\mathrm{x}$

21. Tang Q, Guo W, Zheng L, Wu JX, Liu M, Zhou X, Zhang X, Chen L (2018) Structure of the receptor-activated human TRPC6 and TRPC3 ion channels. Cell Res 28:746-755. https://doi. org/10.1038/s41422-018-0038-2

22. Ong EC, Nesin V, Long CL, Bai CX, Guz JL, Ivanov IP, Abramowitz J et al (2013) A TRPC1 protein-dependent pathway regulates osteoclast formation and function. J Biol Chem 288:22219-22232. https://doi.org/10.1074/jbc.M113.459826

23. Reading SA, Earley S, Waldron BJ, Welsh DG, Brayden JE (2005) TRPC3 mediates pyrimidine receptor-induced depolarization of cerebral arteries. Am J Physiol Heart Circ Physiol 288:H20552061. https://doi.org/10.1152/ajpheart.00861.2004

24. Dietrich A, Chubanov V, Kalwa H, Rost BR, Gudermann T (2006) Cation channels of the transient receptor potential superfamily: their role in physiological and pathophysiological processes of smooth muscle cells. Pharmacol Ther 112:744-760. https://doi. org/10.1016/j.pharmthera.2006.05.013

25. Álvarez-Miguel I, Cidad P, Pérez-García MT, López-López JR (2017) Differences in TRPC3 and TRPC6 channels assembly in mesenteric vascular smooth muscle cells in essential hypertension. J Physiol 595:1497-1513. https://doi.org/10.1113/JP273327 
26. Takayanagi H, Kim S, Koga T, Nishina H, Isshiki M, Yoshida H, Saiura A et al (2002) Induction and activation of the transcription factor NFATc1 (NFAT2) integrate RANKL signaling in terminal differentiation of osteoclasts. Dev Cell 3:889-901. https://doi. org/10.1016/S1534-5807(02)00369-6

27. Trebak M, Vazquez G, Bird GSJ, Putney JW (2003) The TRPC3/6/7 subfamily of cation channels. Cell Calcium 33:451461. https://doi.org/10.1016/S0143-4160(03)00056-3

28. Sel S, Rost BR, Yildirim AO, Sel B, Kalwa H, Fehrenbach K, Renz H et al (2008) Loss of classical transient receptor potential 6 channel reduces allergic airway response. Clin Exp Allergy 38:1548-1558. https://doi.org/10.1111/j.1365-2222.2008.03043 . $\mathrm{X}$

29. Pober BR (2010) Williams-Beuren Syndrome. N Engl J Med 362:239-252. https://doi.org/10.1056/NEJMra0903074

30. Letavernier E, Rodenas A, Guerrot D, Haymann JP (2012) Williams-Beuren syndrome hypercalcemia: is TRPC3 a novel mediator in calcium homeostasis? Pediatrics 129:e1626-1630. https:// doi.org/10.1542/peds.2011-2507

31. Smedlund K, Tano JY, Vazquez G (2010) The constitutive function of native TRPC 3 channels modulates vascular cell adhesion molecule-1 expression in coronary endothelial cells through nuclear factor kappaB signaling. Circ Res 106:1479-1488. https ://doi.org/10.1161/CIRCRESAHA.109.213314

32. White TA, Xue A, Chini EN, Thompson M, Sieck GC, Wylam ME (2006) Role of Transient Receptor Potential C3 in TNF- $\alpha-$ Enhanced Calcium Influx in Human Airway Myocytes. Am J Respir Cell Mol Biol 35:243-251. https://doi.org/10.1165/ rcmb.2006-0003OC

33. Becker EB, Oliver PL, Glitsch MD, Banks GT, Achilli F, Hardy A, Nolan PM et al (2009) A point mutation in TRPC3 causes abnormal Purkinje cell development and cerebellar ataxia in moonwalker mice. Proc Natl Acad Sci USA 106:6706-6711. https ://doi.org/10.1073/pnas.0810599106

34. Weycker D, Li X, Barron R, Bornheimer R, Chandler D (2016) Hospitalizations for osteoporosis-related fractures: economic costs and clinical outcomes. Bone Rep 5:186-191. https://doi. org/10.1016/j.bonr.2016.07.005

35. Morse LR, Battaglino RA, Stolzmann KL, Hallett LD, Waddimba A, Gagnon D, Lazzari AA et al (2009) Osteoporotic fractures and hospitalization risk in chronic spinal cord injury. Osteoporos Int 20:385-392. https://doi.org/10.1007/s00198-008-0671-6

36. Jennings LA, Auerbach AD, Maselli J, Pekow S, Lindenauer PK, Lee SJ (2010) Missed opportunities for osteoporosis treatment in patients hospitalized for hip fracture. J Am Geriatr Soc 58:650 657. https://doi.org/10.1111/j.1532-5415.2010.02769.x

37. Reyes C, Hitz M, Prieto-Alhambra D, Abrahamsen B (2016) Risks and Benefits of Bisphosphonate therapies. J Cell Biochem 117:20-28. https://doi.org/10.1002/jcb.25266

38. Black DM, Delmas PD, Eastell R, Reid IR, Boonen S, Cawley JA, Cosman F et al (2007) Once-yearly zoledronic acid for treatment of postmenopausal osteoporosis. N Engl J Med 356:1809-1822. https://doi.org/10.1056/NEJMoa067312

39. Cummings SR, San Martin J, McClung MR, Siris ES, Eastell R, Reid IR, Delmas P et al (2009) Denosumab for prevention of fractures in postmenopausal women with osteoporosis. N Engl J Med 361:756-765. https://doi.org/10.1056/NEJMoa0809493

40. Karatoprak C, Kayatas K, Kilicaslan H, Yolbas S, Yazimci NA, Gümüskemer T, Demirtunç R (2012) Severe hypercalcemia due to teriparatide. Indian J Pharmacol 44:270-271. https://doi. org/10.4103/0253-7613.93869

41. Kyrgidis A, Toulis KA (2011) Denosumab-related osteonecrosis of the jaws. Osteoporos Int 22:369-370. https://doi.org/10.1007/ s00198-010-1177-6

42. Tiapko O, Groschner K (2018) TRPC3 as a target of novel therapeutic interventions. Cells 7:83. https://doi.org/10.3390/cells 7070083

43. Kiyonaka S, Kato K, Nishida M, Mio K, Numaga T, Sawaguchi Y, Yoshida T et al (2009) Selective and direct inhibition of TRPC3 channels underlies biological activities of a pyrazole compound. Proc Natl Acad Sci USA 106:5400-5405. https://doi.org/10.1073/ pnas.0808793106

Publisher's Note Springer Nature remains neutral with regard to jurisdictional claims in published maps and institutional affiliations. 